\title{
A Chinese Chan-based lifestyle intervention improves memory of older adults
}

\author{
Agnes S. Chan ${ }^{1,2,3}$, Sophia L. Sze ${ }^{1,2}$, Jean Woo and Ruby H. Yu ${ }^{4}$ \\ 1 Department of Psychology, The Chinese University of Hong Kong, New Territories, Hong Kong, China \\ ${ }^{2}$ Chanwuyi Research Center for Neuropsychological Well-Being, The Chinese University of Hong Kong, New Territories, Hong Kong, China \\ ${ }^{3}$ Henan Songshan Research Institute for Chanwuyi, Zheng Zhou, China \\ ${ }^{4}$ Department of Medicine and Therapeutics, The Chinese University of Hong Kong, New Territories, Hong Kong, China
}

\section{Edited by:}

Rodrigo Orlando Kuljiš, Zdrav Mozak Limitada, Chile

Reviewed by:

Elizabeta Blagoja

Mukaetova-Ladinska, Newcastle

University, UK

Guo Qihao, Huashan Hospital - Fudan

University, China

\section{*Correspondence:}

Agnes S. Chan, Department of Psychology, The Chinese University of Hong Kong, Room 355, 3/F, Sino Building, Shatin, New Territories, Hong Kong, China

e-mail: aschan@psy.cuhk.edu.hk
This study aims to explore the potential benefits of a Chinese Chan-based lifestyle intervention on enhancing memory in older people with lower memory function. Fortyfour aged 60-83 adults with various level of memory ability participated in the study. Their memories (including verbal and visual components) were assessed before and after 3 months intervention. The intervention consisted of 12 sessions, with one 90 min session per week. The intervention involved components of adopting a special vegetarian diet, practicing a type of mind-body exercises, and learning self-realization. Elderly with lower memory function at the baseline (i.e., their performance on standardized memory tests was within 25th percentile) showed a significant memory improvement after the intervention. Their verbal and visual memory performance has showed 50 and $49 \%$ enhancement, respectively. In addition, their improvement can be considered as a reliable and clinically significant change as reflected by their significant pre-post differences and reliable change indices. Such robust treatment effect was found to be specific to memory functions, but less influencing on the other cognitive functions. These preliminary encouraging results have shed some light on the potential applicability of the Chinese Chan-based lifestyle intervention as a method for enhancing memory in the elderly population.

Keywords: memory, lifestyle intervention, Chinese, elderly, mind-body exercise, diet

\section{INTRODUCTION}

Mild cognitive impairment (MCI), also known as isolated memory impairment, is a brain disorder primarily affects memory. While the older adults may begin to forget important information such as appointments or conversation, this impairment does not interfere with their daily activities (Petersen et al., 1999). Empirical evidence has showed that MCI is a risk factor for later progression to dementia. That is, studies have suggested that these individuals tend to progress to probable Alzheimer's disease $(\mathrm{AD})$ at a rate of approximately 10-15\% per year (Bowen et al., 1997; Petersen et al., 1999, 2001). Scientific reviews have suggested that various cognitivebased interventions for $\mathrm{AD}$ type of dementia are rather labor intensive, yet with limited efficacy, particularly in managing the core symptom of memory deficits (Clare and Woods, 2004; Choi and Twamley, 2013). Therefore, some research efforts have been directed to explore effective preventive measures for normal elderly so as to reduce their risk of developing dementia. Because memory impairment is a relatively important pre-clinical feature of dementia, vigorous research efforts have focused on exploring methods to delay the decline or enhance the memory function in normal elderly (Rasmunsson et al., 1999; Mahncke et al., 2006; Papp et al., 2009; Smith et al., 2009; McAvinue et al., 2013). Nevertheless, as suggested by a recent review (Papp et al., 2009), the efficacy of various cognitive interventions for healthy elderly is rather limited. Some interventions have no significant effect (Rasmunsson et al., 1999; Edwards et al., 2002); others showed very minimal memory enhancement of $<10 \%$ (at small effect size; Mahncke et al., 2006;
Willis et al., 2006; McAvinue et al., 2013). For instance, Smith et al. (2009) reported results of an intensive computerized training (five $1 \mathrm{~h}$ sessions per week for 8 weeks) with approximately $10 \%$ postintervention increment in delayed recall performance of elderly subjects.

Besides cognitive training, another emerging line of research has proposed lifestyle intervention for mental and physical wellness of the elderly. Lifestyle intervention, an integrative intervention comprising diet, exercise, and at least one other component (e.g., stress management, counseling, or smoking cessation), aims to reverse pathology and/or delay disease progression by modifying high-risk health habits (Lifestyle Medicine, 2013). Some research findings have supported the effects of certain lifestyle factors or some comprehensive lifestyle intervention programs in improving mental (e.g., depressive mood), and physical wellness (e.g., blood pressure level) of aging people (Chan et al., 2005; Dickinson et al., 2006; Witte et al., 2009; Ruscheweyh et al., 2011; Clark et al., 2012; McGregor et al., 2013). Nevertheless, the effect of lifestyle intervention on memory problem is relatively unclear.

Given the potential efficacy and easy accessibility of lifestyle intervention, this study aimed to explore the effect of a Chinese Chan-based lifestyle intervention, the Dejian Mind-Body Intervention (DMBI; Chan, 2010, 2013), on improving the memory function of community-dwelling elderly people. This intervention was developed upon the medical principles originating from the Shaolin Temple. DMBI, a holistic approach facilitating better 
well-being and quality of life through modification of one's life habits, comprises diet modification, mind-body exercises, and self-realization. Cumulative empirical and clinical evidence has supported the positive effects of DMBI on cognitive functions, mood, problem behaviors, and physical health of healthy adults and patients with various brain disorders (Chan et al., 2008, 2009, 2011 a,b,c, 2012a,b,c, 2013a,b, 2014). A randomized controlled study has found significantly improved attention and depressive mood (Chan et al., 2012a), and physical condition and sleep problem (Chan et al., 2012b) in patients with depression. Their enhancement in cognitive functions was associated with their altered brain electrophysiological activity pattern (Chan et al., 2013b). Other studies also showed improvement on various cognitive functions including attention, memory, self-control, and executive functions on children with autism (Chan et al., 2008, 2011a, 2012c, 2013a, 2014). Furthermore, some pilot data have suggested positive effects of DMBI on enhancing psychological and physical wellness of community-dwelling elderly (Yu et al., in press). Given the encouraging results on individuals with depression and autism and some preliminary evidence for elderly people, the present study extends the previous findings and examine if this intervention method can have positive effect on older adults with lower level of memory function. A group of independently living elders was recruited, and about 30\% of them demonstrated certain degree of memory problems as suggested by their relatively poorer performance (at or below 25th percentile) on the standardized memory tests. The primary purpose of the present study is to examine if a Chinese Chan-based intervention can improve the memory function of this group of older adults.

\section{MATERIALS AND METHODS PARTICIPANTS}

Forty-four participants aged 60-83 were recruited from six community health and social centers in the New Territories East regions in Hong Kong and through Internet publicity. Participants with a history of head injury or neurological/psychiatric disorders, who could not walk, or had severe illnesses, were excluded. They were blind to the potential benefits of the intervention. The attrition rate was $5 \%$, and the mean attendance rate was 93\%. The participants were divided into three groups according to their performance on the standardized verbal and visual memory tests: (1) high performers: score at or above 75 th percentile; (2) medium performers: score between 26th and 74th percentile; (3) low performers: score at or below 25th percentile. The Hong Kong List Learning Test (HKLLT) and the Visual Reproduction subtest of the Wechsler Memory Scale III (WMS-VR) are selected as the tests for verbal and visual memory function and the classification of the three groups of performers are based on the performance of delayed recall in these two memory tests. The reason for choosing delayed recall performance is that it bears $90 \%$ accuracy in predicting the progression from MCI to dementia after 2 years (Tierney et al., 1996). In particular, the delayed recall in verbal learning test is one of the neuropsychological assessment indices that commonly used to measure memory decline associated with aging and MCI (Hulette etal., 1998; Artero etal., 2003; Gauthier et al., 2006) and the most accurate cognitive measure in discriminating between individuals with presymptomatic $\mathrm{AD}$ and those who remained without dementia (Chen et al., 2000).

Table 1 presents the demographic characteristics of the three groups for the verbal and visual memory function. The global cognitive function of each participant was assessed by the Chinese version of Mattis Dementia Rating Scale (CDRS; Chan et al., 2003a) with a purpose to screen out participant having dementia. As revealed by their performance on the CDRS, all participants scored above the optimal cutoff score of the adjusted total score (i.e., 141), which suggested that none of them had dementia and they demonstrated intact global cognitive functions. In general, all three groups of performers had comparable age, education, gender, handedness, CDRS adjusted score, blood pressure level, and depressive mood problems (as measured by the Chinese Geriatric Depression Scale-short form (CGDS-SF; Lee et al., 1993), F ranges from $0.07-3.05, p>0.05$, except for the groups of visual memory function that the high performers were younger than the medium performers, $p=0.032$, the low performers had lower CDRS and CGDS-SF score than medium performers, $p=0.006$ and 0.027 , and the low performers had higher systolic blood pressure level than high performers, $p=0.019$.

\section{PROCEDURE}

The study was conducted in accordance with the Helsinki Declaration of the World Medical Association Assembly. The research protocol was approved by the Joint Chinese University of Hong Kong - New Territories East Cluster Clinical Research Ethics Committee. All participants voluntarily participated in the study, and their informed consent was obtained prior to the study. Research assistants who were blind to the experimental design and potential effects of the intervention individually assessed the demographic information, medical history, and cognitive functions of each participant before and after intervention. After the baseline assessment, the participants attended group training on the Chinese Chan-based lifestyle intervention for 12 weekly $90 \mathrm{~min}$ sessions.

\section{INTERVENTION}

The DMBI is a Chinese Chan-based lifestyle intervention based on the Chanwuyi tradition (i.e., Zen, martial arts and healing) and the Chan medical principle. According to the Chan medical principle, majority of mental and physical health problems are due to the blockage of orifices (i.e., bodily openings) and stagnation of Qi and blood circulation, which are likely resulted from unhealthy living style and attitude. Thus, adoption of a healthy lifestyle and positive thinking is a key to unblock the orifices and smooth the Qi and blood circulation, so as to enhance health. Emphasizing integrative treatment of the mind and the body, the principle of DMBI is to enhance mental and physical health by changing daily dietary and exercise habits and to improve the psychological wellbeing by understanding the root of problems in accordance with Buddhist philosophy (Chan, 2010, 2013).

The intervention was conducted by a clinical psychologist who is familiar with the DMBI model. Throughout the 12 training sessions, the participants were taught the fundamental principles and techniques of DMBI, and their progress was closely monitored by 
Table 1 | Demographic characteristics of participants.

\begin{tabular}{|c|c|c|c|c|c|c|c|c|c|c|}
\hline Characteristics & $\begin{array}{l}\text { LP } \\
(n=18)\end{array}$ & $\begin{array}{l}\text { MP } \\
(n=9)\end{array}$ & $\begin{array}{l}\text { HP } \\
(n=14)\end{array}$ & $F$ or $\chi^{2}$ & $p$ & $\begin{array}{l}\text { LP } \\
(n=10)\end{array}$ & $\begin{array}{l}\text { MP } \\
(n=21)\end{array}$ & $\begin{array}{l}\text { HP } \\
(n=11)\end{array}$ & $F$ or $\chi^{2}$ & $\boldsymbol{p}$ \\
\hline Age (years) & $\begin{array}{l}65.94 \\
(5.54)\end{array}$ & $\begin{array}{l}66.56 \\
(5.81)\end{array}$ & $\begin{array}{l}64.00 \\
(2.86)\end{array}$ & 0.95 & 0.40 & $\begin{array}{l}65.50 \\
(4.84)\end{array}$ & $\begin{array}{l}66.76 \\
(5.37)\end{array}$ & $\begin{array}{l}62.27 \\
(1.90)\end{array}$ & 3.46 & $0.04 *$ \\
\hline Gender (male:female) & $12: 6$ & $5: 4$ & $5: 9$ & 3.05 & 0.22 & $5: 5$ & $12: 9$ & $6: 5$ & 0.14 & 0.93 \\
\hline Handedness (right-handed \%) & $16: 2$ & $9: 0$ & $13: 1$ & 1.09 & 0.58 & $10: 0$ & $20: 1$ & $9: 2$ & 2.97 & 0.23 \\
\hline CDRS (adjusted total score) & $\begin{array}{l}165.64 \\
(4.75)\end{array}$ & $\begin{array}{l}168.86 \\
(4.74)\end{array}$ & $\begin{array}{l}167.52 \\
(3.33)\end{array}$ & 2.28 & 0.12 & $\begin{array}{l}163.76 \\
(5.76)\end{array}$ & $\begin{array}{l}168.74 \\
(3.49)\end{array}$ & $\begin{array}{l}166.60 \\
(2.52)\end{array}$ & 4.83 & $0.01 *$ \\
\hline CGDS-SF & $\begin{array}{l}2.39 \\
(3.11)\end{array}$ & $\begin{array}{l}2.00 \\
(1.80)\end{array}$ & $\begin{array}{l}2.29 \\
(2.27)\end{array}$ & 0.07 & 0.94 & $\begin{array}{l}4.10 \\
(3.54)\end{array}$ & $\begin{array}{l}1.67 \\
(1.59)\end{array}$ & $\begin{array}{l}1.82 \\
(2.27)\end{array}$ & 3.93 & $0.03 *$ \\
\hline
\end{tabular}

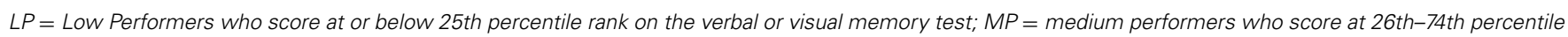

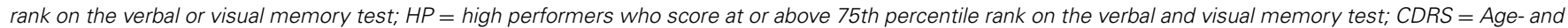

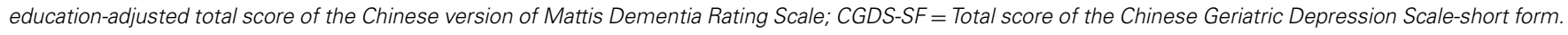
${ }^{*} p<0.05$.

the trainer. There were three intervention components: (1) Advise diet modification by reducing intake of food (e.g., ginger, garlic, green onions, spicy foods, eggs, meat, and fish) that generates excessive internal heat and adversely affects moods and physical health based on Chan medical principle; and consuming food that was good for health everyday (e.g., fresh vegetables, fruits, grains, beans, mushrooms, nuts, and root vegetables). (2) Practice Nei Gong, which is mind-body exercise, composing sets of breathing exercises and gentle and calm movement. For instance, rolling the hands slowly up and down between the chest and the abdomen, resting the hands on the abdomen while quietly observing the breathing. Regular practice of Nei Gong helps reducing stress, and improving overall physical health and the circulation of Qi and the blood. The basic principles and demonstration of Nei Gong have been elaborated on our website (www.chanwuyicenter.com) and in two published books (Chan, 2010, 2013). The practice time was not fixed, and participants were instructed to practice the exercises until they felt warm and relaxed. (3) Improving psychological wellbeing by increasing awareness and sensitivity to how unrealistic desires (i.e., greed), anger, and obsession (i.e., unrealistic craving for something or somebody) affect mental and physical health, and modifying thought process to alleviate excessive desires, anger, and obsessions.

According to the log record of home practice, 60\% participants abstained from or reduced their intake of the "not recommended" food, and $81 \%$ participants consumed at least three "recommended" food categories every day. Also, 95\% participants practiced Nei Gong every day for an average of about $25 \mathrm{~min}$ per day.

\section{MEASURES}

\section{Hong Kong List Learning Test}

The HKLLT is a well-standardized test of verbal memory in the Chinese population (Chan et al., 2003b; Chan, 2006). Participants were required to learn a list of 16 Chinese words through three learning trials. After $30 \mathrm{~min}$ delay, they were asked to recall as many words as possible. The total number of recalled words after a delay was used to reflect verbal memory ability.

\section{Visual reproduction subtest of the Wechsler Memory Scale III}

The VR is a common test for visual memory function (Wechsler, 2005). Participants were required to learn five sets of geometric forms, and then draw them from memory after $30 \mathrm{~min}$. The total score in terms of accuracy rate at a delayed recall was adopted for analyses. In addition, the total score at Copy trial, which simply required the participant to copy the geometric forms on the paper, was used to reflect the ability to construct visual forms, and to control the possible influence of deficient drawing ability on recall performance. It should be noted that the lowest Copy score in present study is 90 out of 104 (accuracy rate $=87 \%$ ), which means that all participants have intact drawing ability and thus poor recall performance, if any, is unlikely due to deficient drawing ability.

\section{Chinese Version of Mattis Dementia Rating Scale}

The CDRS is a locally validated test that was culturally adapted from the Mattis Dementia Rating Scale to measure general cognitive functions, as indicated by its education- and age-adjusted total score, of the elderly people (Chan et al., 2001, 2003a). This scale was found to have good reliability with internal consistency 
ranging from $0.7-0.9$, and satisfactory construct validity (Chan et al., 2001). It consists of 36 tasks in five subscales, including Attention, Memory, Conceptualization, Initiation/Perseveration (CDRS-I/P), and Construction, which tap abilities of visual and auditory attention and memory, abstraction, flexible cognitive control and visuospatial construction, respectively. The maximum total raw score of the scale is 144 . However, the adjusted total score, instead of the total raw score, is adopted so as to control the confounding effect of age and education. Adjusted total score that is below the optimal cutoff score of 141 suggests higher probability of having dementia.

\section{Color Trail Test}

The Color Trail Test (CTT) is a standardized test for testing flexible set-shifting behavior (D'Elia et al., 1996). The total time (s) to complete the second trial of the CTT (CTT-T2), which requires connecting numbers in correct sequence while alternating between two colors embedding the numbers, was used to reflect degree of flexibility in mental shift.

\section{Category Fluency Test}

The Category Fluency Test (CFT) is a test for language production and fluency of speech (Chan and Poon, 1999). It requires generation of exemplars of two semantic categories, namely animals and transportation, as many as possible within time constraint. The total number of items generated was used to measure degree of fluent speech production.

\section{STATISTICAL ANALYSIS}

The statistical package for the social sciences (SPSS; version 15.0) was used for data analysis. Repeated measures of analysis of variance (ANOVA) and post hoc paired $t$-tests were used to evaluate the changes across the three groups of performers. The extent of pre-post difference was evaluated with effect size. Additionally, the reliable change index (RCI) was computed to examine the clinical significance and reliability of the intervention effect. The RCI is calculated by dividing the pre-post difference score by the standard error of difference between the two test scores $\left(R C I=\frac{x 2-x 1}{\text { Sdiff }}\right)$ (Jacobson and Truax, 1991). As proposed by Wise (2004), a clinically significant and reliable change can be reflected by a pre-post difference scores of $>0.5 \mathrm{SD}$ and a RCI $>0.84 . \chi^{2}$ statistics was adopted to compare number of participants with or without a clinically significant and reliable change in memory performance.

\section{RESULTS \\ GREATER ENHANCEMENT IN MEMORY FUNCTION OF LOW-PERFORMING ELDERLY AFTER INTERVENTION}

Comparisons on the change in memory performance across three groups of elderly having different baseline levels of memory functioning revealed that elderly with lower performance level at baseline demonstrated greater extent of enhancement after intervention. Results of repeated measures ANOVA showed that there were significant Time (Pre vs. Post) by Group (three subgroups) interaction effect for both HKLLT, a measure of verbal memory, $F(2,38)=3.58, p=0.038$, and VR, a measure of visual memory, $F(2,39)=4.41, p=0.019$. Table 2 presents the pre-post changes across the three groups analyzed by post hoc $t$-test. It was found that the low performers showed a significant improvement in both verbal, $t=-6.89$, $p=0.00$, effect size $=1.62$, and visual memory, $t=-2.80$, $p=0.01$, effect size $=0.88$, with an extent of 50 and $49 \%$

Table 2 | Memory performance of the three groups of performers at pre- and post-intervention.

\begin{tabular}{|c|c|c|c|c|c|c|}
\hline & Pre & Post & Difference & $t$ value & $p$ value & E.S. \\
\hline \multicolumn{7}{|l|}{ Low performers } \\
\hline \multirow{2}{*}{ Verbal memory $(n=18)$} & 6.06 & 9.06 & 3.00 & -6.89 & $0.00 * *$ & $1.62++$ \\
\hline & $(1.26)$ & $(1.80)$ & $(1.85)$ & & & \\
\hline \multirow[t]{2}{*}{ Visual memory $(n=10)$} & 35.10 & 52.40 & 17.30 & -2.80 & $0.01 *$ & $0.88++$ \\
\hline & $(9.72)$ & $(21.51)$ & (19.56) & & & \\
\hline \multirow[t]{2}{*}{ Verbal memory $(n=9)$} & 8.89 & 9.89 & 1.00 & -0.97 & 0.18 & 0.32 \\
\hline & $(0.93)$ & (3.10) & (3.08) & & & \\
\hline \multirow[t]{2}{*}{ Visual memory $(n=21)$} & 57.90 & 74.05 & 16.14 & -5.70 & $0.00^{* *}$ & $1.24++$ \\
\hline & (8.68) & $(9.91)$ & (12.99) & & & \\
\hline \multicolumn{7}{|l|}{ High performers } \\
\hline Visual memory $(n=11)$ & $(5.20)$ & $(14.02)$ & (13.27) & & & \\
\hline
\end{tabular}

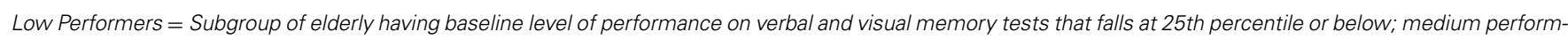

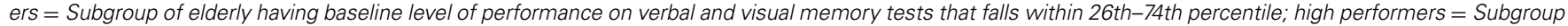

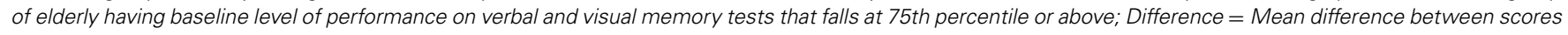
at pre- and post-intervention; E.S. = Effect size. Standard deviations are in parenthesis. ${ }^{*} p<0.05,{ }^{* *} p<0.01$ at one-tailed; ++ large effect size. 


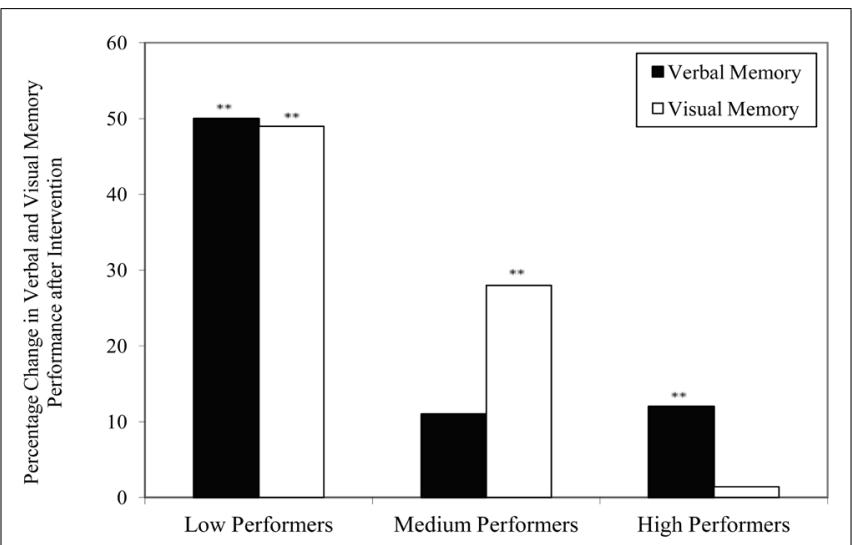

FIGURE 1 | Percentage change in raw scores of verbal and visual memory tests across three levels of performance subgroups after intervention. ${ }^{* *} p<0.01$ in paired samples $t$-test (one-tailed).

improvement, respectively (Figure 1). However, the extent of score-increment in medium and high performers was to a lesser extent (Table 2), which ranges from 1.4-28\% (Figure 1). It should be noted that the extent of memory enhancement in low performers was significantly greater than that of the medium (HKLLT: $p=0.006$ ) and high performers (HKLLT: $p=0.002$; VR: $p=0.006)$.

The scatter-plots in Figures 2A,B showed a negative association between the baseline level of performance on verbal and visual memory and the percentage change in respective test performance after intervention, which was statistically significant, $r=-0.61$ and $-0.57, p=0.00$. It suggests that elderly individuals with lower baseline level of memory function tend to demonstrate greater extent of memory enhancement after intervention, as compared to those having higher functioning level at baseline. These results implicate that the Chan-based lifestyle intervention seems to have positive effects on enhancing memory functions of older people who have relatively poorer memory.

\section{MEMORY ENHANCEMENT IN LOW PERFORMERS IS RELIABLE AND CLINICALLY SIGNIFICANT}

In order to determine if the intervention effect on memory function is a reliable and clinically significant change, the Low Performers were further classified into either "Improved", "No Change," or "Declined" categories based on the criteria of pre-post change in test scores and RCI suggested by Wise (2004), which has been defined in Table 3. It was found that $72 \%$ participants demonstrated a clinically significant and reliable increase in verbal memory performance (i.e., "Improved" cluster), which was significantly more than the proportion of participants classified into the other two clusters, $\chi^{2}(2)=14.33, p<0.001$. A similar result was found for visual memory performance, where $60 \%$ participants demonstrated clinically significant memory enhancement, though such distribution did not reach statistical significance, $\chi^{2}(2)=3.80$, $p=0.075$. Therefore, the memory enhancement effect of DMBI for the elderly participants with a lower level of memory function was clinically significant and reliable. It suggests
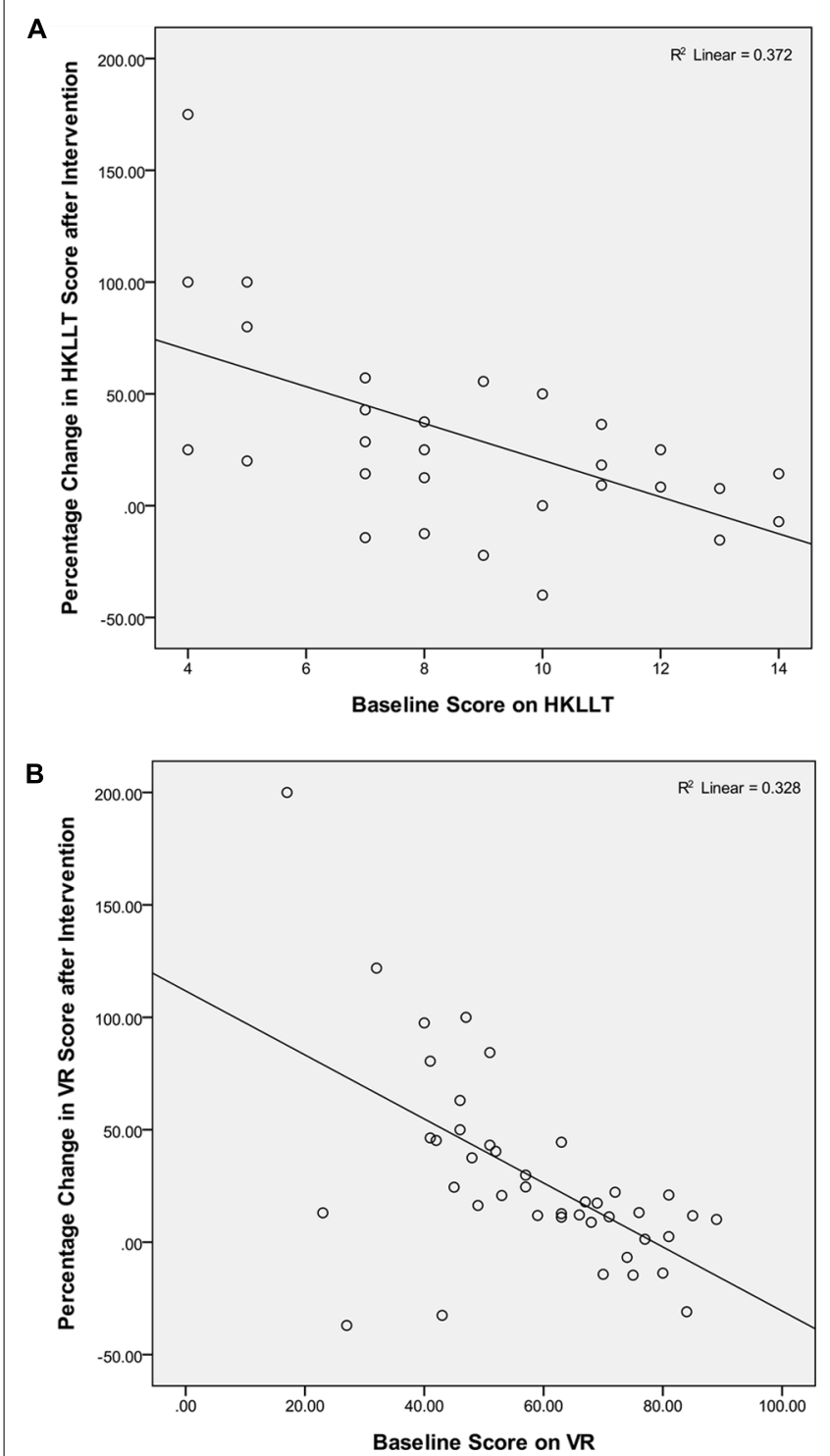

FIGURE 2 | Association between baseline level of performance on (A) verbal and $(B)$ visual memory tests and the percentage change in test performance after intervention.

that the Chan-based lifestyle intervention might be an effective memory intervention for older people with poorer memory performance.

\section{SPECIFIC TREATMENT EFFECT ON MEMORY FUNCTIONS OF OLDER PEOPLE}

To explore whether the positive treatment outcome was specifically for memory functions or the treatment would also benefit the other cognitive aspects of the older people, pre-post comparisons on the other cognitive functions based on the whole group was performed with paired samples $t$-tests. Table 4 presents the performance of the participants on various cognitive tests before and after intervention. It was found that the most robust treatment effect was still in verbal and visual memory function. In 
Table 3 | Distribution of participants in the low performers with and without clinically significant changes in memory performance after intervention.

\begin{tabular}{llll}
\hline & $\boldsymbol{N}$ & $\boldsymbol{\chi}^{\mathbf{2}}(\mathbf{d f})$ & $\boldsymbol{p}$ value \\
\hline $\begin{array}{l}\text { Verbal memory }(n=18) \\
\quad \text { Improved }\end{array}$ & 13 & $14.33(2)$ & $0.00^{* *}$ \\
$\quad$ No Change & 5 & & \\
Declined & 0 & & \\
Visual memory $(n=10)$ & & $3.80(2)$ & 0.075 \\
Improved & 6 & & \\
No change & 3 & & \\
Declined & 1 &
\end{tabular}

Improved $=$ pre-post change in test scores $>+0.5 \mathrm{SD}$ and $\mathrm{RCl}>+0.84$; No change $=$ pre-post change in test scores in-between $-0.5 S D$ and $+0.5 S D$ and $-0.84<R C l<+0.84 ;$ Declined $=$ pre-post change in test scores $>-0.5 S D$ and $\mathrm{RCl}>-0.84 ; * * 0<0.01$ at one-tailed.

the verbal memory test, there was a significant mean increment of two words recalled after intervention, $t=-5.81, p=0.00$, effect size $=0.91$, with an extent of $23 \%$ improvement. A similar degree of enhancement was found in visual memory test, which showed a significant 12-point increment in delayed recall score after intervention, $t=-5.05, p=0.00$, effect size $=0.78$, with an extent of $21 \%$ improvement. Although there was a significant improvement in the cognitive tests that measures ability to flexibly shifting mental set (CDRS-I/P and CTT-T2), $t=-2.76$ and 3.55, $p=0.01$ and 0.00 , respectively, their extent of improvement was to a much lesser degree (CDRS-I/P: 3\%; CTT-T2: 12\%). Furthermore, as expected, some other relatively more crystallized cognitive ability, such as language, and the cognitive abilities that are less susceptible to aging-related cognitive decline, such as visuospatial construction, did not show any significant changes after intervention, $t$ ranges from -1.0 to $1.95, p>0.05$. In summary, the results suggested that the Chan-based lifestyle intervention seemed to have memory-specific positive effects for older people.

\section{DISCUSSION}

The findings of the present study provide preliminary support for the effects of a 3 months Chinese Chan-based lifestyle intervention in improving memory function of older adults with poorer memory performance. The extent of increment in delayed recall score (for 50\%, at large effect size) on both verbal and visual memory in present study was very encouraging. Such improvement met the criteria of clinical significance and reliable change, suggesting that the improvement is likely treatment-specific and probably not due to practice effect. The previous studies on various cognitive training (Mahncke et al., 2006; Willis et al., 2006; Smith et al., 2009) and lifestyle intervention (Witte et al., 2009; Ruscheweyh et al., 2011) reported some positive effects on memory function that is consistent with the present study, suggesting that memory ability can be improved with appropriate intervention. However, the effects of the present intervention seem to be to a greater extent compared to the conventional cognitive training
Table 4 | Changes in various cognitive functions before and after intervention.

\begin{tabular}{lllllll}
\hline & Pre & Post & Difference & $\boldsymbol{t}$ & $\boldsymbol{p}$ & E.S. \\
\hline Memory & & & & & & \\
HKLLT-DR & 8.73 & 10.76 & 2.02 & -5.81 & $0.00^{* *}$ & $0.91++$ \\
& $(2.89)$ & $(2.85)$ & & & & \\
WMS-VR-DR & 58.12 & 70.60 & 12.48 & -5.05 & $0.00 * *$ & $0.78+$ \\
& $(17.76)$ & $(17.63)$ & & & &
\end{tabular}

\begin{tabular}{lcccccc} 
Attention & & & & & & \\
CDRS-Att & 36.43 & 36.57 & 0.14 & -0.78 & 0.22 & 0.12 \\
& $(1.17)$ & $(0.67)$ & & & & \\
\multicolumn{7}{l}{ Cognitive flexibility } \\
CDRS-I/P & 35.43 & 36.40 & 0.98 & -2.76 & $0.00 * *$ & 0.43 \\
& $(2.48)$ & $(1.15)$ & & & & \\
CTT-T2\# & 109.66 & 96.88 & -12.78 & 3.55 & $0.00 * *$ & $0.55+$ \\
& $(40.39)$ & $(37.99)$ & & & &
\end{tabular}

\begin{tabular}{|c|c|c|c|c|c|c|}
\hline \multicolumn{7}{|c|}{ Conceptualization } \\
\hline \multirow[t]{2}{*}{ CDRS-concept } & 37.67 & 37.74 & 0.07 & -0.24 & 0.41 & 0.04 \\
\hline & $(1.98)$ & $(1.95)$ & & & & \\
\hline \multicolumn{7}{|l|}{ Language } \\
\hline \multirow[t]{2}{*}{ CFT } & 29.17 & 29.86 & 0.69 & -0.93 & 0.18 & 0.14 \\
\hline & $(5.21)$ & $(4.42)$ & & & & \\
\hline \multicolumn{7}{|c|}{ Visuospatial construction } \\
\hline \multirow[t]{2}{*}{ CDRS-construct } & 5.93 & 5.98 & 0.05 & -1.0 & 0.16 & 0.15 \\
\hline & $(0.26)$ & $(0.15)$ & & & & \\
\hline \multirow[t]{2}{*}{ WMS-VR-copy } & 98.90 & 98.19 & -0.71 & 1.95 & $0.03 *$ & 0.30 \\
\hline & $(3.06)$ & (3.19) & & & & \\
\hline
\end{tabular}

$H K L L T-D R=$ Delayed recall score of the Hong Kong List Learning Test; WMS-VR$D R=$ Delayed recall score in the Visual Reproduction subscale of the Wechsler Memory Scale-III; Chinese version of Mattis Dementia Rating Scale CDRSAtt $=$ Attention subscale score of the CDRS-I/P = Initiation/Perseveration subscale score of the CDRS; CTT-T2 = Completion time of the Color Trail Test-Trial 2; CDRS-Concept $=$ Conceptualization subscale score of the CDRS; CFT = Category Fluency Test; $C D R S$-Construct = Construction subscale score of the CDRS; WMS$V R$-Copy $=$ Copy score in the VR of the WMS-III; Difference $=$ Post-intervention score minus pre-intervention score; E.S. = Effect size. Standard deviations are in parenthesis. "Lower value indicates better performance; ${ }^{*} p<0.05,{ }^{* *} p<0.01$; + medium effect size, ++large effect size.

program and other lifestyle interventions. For instance, 3 month caloric restriction can trigger a $20 \%$ increment in delayed recall (Witte et al., 2009), 6 month low-intensity aerobic exercise training can induce a $10 \%$ increase in immediate recall (Ruscheweyh et al., 2011), and present results demonstrated a 50\% improvement on delayed recall. It should also be noted that the present study recruited elderly with isolated memory difficulty while the other studies did not target older adults with memory difficulty. Thus, the memory-enhancing effect of the Chan-based lifestyle intervention has implicated its possibility as a method in facilitating memory function in older adults or even individuals with MCI.

The present result was consistent with our previous studies on other clinical populations (Chan et al., 2008, 2009, 2011a,b,c, 
2012a,b,c, 2013a,b, 2014). In a series of studies on autism, the participants demonstrated significant improvement on cognitive function after 1 month intervention on changing diet (Chan et al., 2012c) and practicing Nei Gong (Chan et al., 2013a). In addition, an autistic child with moderately-severely impaired memory ability was found to have improved up to normal low-average range after an 8 month Chan-based intervention (Chan et al., 2011a). Similarly, randomized controlled studies on patients with clinical depression showed significant improvement on attention (Chan et al., 2012a) and brain activity pattern (Chan et al., 2013b). Thus, the results of the present study further suggest that this intervention is not only applicable to children and adults but also to elderly people. One reason for the generalization of this intervention is that it involves easy and concrete procedure, and thus even individuals with limited intelligence, and ability are able to follow.

The low attrition rate of $5 \%$ hinted that the Chinese Chan-based lifestyle intervention was very well received by the elderly Chinese participants. This intervention has several merits that can provide clues to this phenomenon. First, the intervention techniques are simple and clearly comprehensible to older adults, e.g., what to eat and what not to eat, and the simple and slow movements of the mind-body exercises. This characteristic is particularly important for the local elderly population, as majority of them are illiterate or have less than 12 years of formal education. Second, as a lifestyle intervention, the treatment components are more accessible and applicable to daily home practice. While the majority of conventional cognitive training methods involve computerized programs, the Chan-based lifestyle intervention requires no special equipment, and only needs limited space for practicing the mind-body exercises, which seems to be more practical and feasible for elderly people of lower socioeconomic status or with limited computer knowledge. Third, this intervention has relatively less restriction in treatment regimen as compared to some other intervention methods. The participants were only recommended to modify their diet and practice the mind-body exercises according to their own lifestyles and plans. The mind-body exercise practice time of the participants averaged $25 \mathrm{~min}$ per day (range 5-60 $\mathrm{min}$ ), and it can be broken down into smaller periods for shorter practice (e.g., $10 \mathrm{~min}$ each time, thrice per day). Such flexibility was not physically demanding for the elderly participants. Finally, the intervention can be conducted on a large scale and requires no in-depth analysis of the personal background and psychological distress of the elderly participants. Therefore, older people, especially the more conservative and traditional Chinese elderly, would feel more comfortable in participating, and the intervention can be run in community centers or homes for the elderly.

Despite these encouraging findings, further verification of the treatment effects on elderly participants with a larger sample size in randomized controlled trials is warranted. Furthermore, the participants in present study are cognitively intact as revealed by their relatively high CDRS score and their memory functions are within normal range, whether the memory-enhancement effect can also be found for elderly people with significant memory deficits or even with dementia is worth investigating. This study focused mainly on the effects on memory function, its effect on the mental and physical domains of the aging population deserves further investigation. In addition, whether this intervention can be applied to non-Chinese population remains unclear and needs further investigation.

\section{ACKNOWLEDGMENTS}

This work was supported by the Health and Health Services Research Fund (grant number 10110931) established by the Food and Health Bureau of the Hong Kong SAR.

The authors would like to specially thank Venerable Master Dejian of the Songshan monastery for his teaching and support. They are also grateful to Ms. Winnie Yeung, Ms. Fannie Yeung, Mr. Moses Wong, Ms. Maggie Ng, Ms. Karoline Chiu, Ms. Hannah Li, and Ms. Carman Chu for their effort in subject recruitment, data collection/management/analysis, and/or literature review. Further appreciation is extended to all participants and to the community health, and social centers (The Centre for Nutritional Studies, Jockey Club, Centre for Osteoporosis Care and Control, Sheen Hok Charitable Foundation, Kwan Shon Hing Yu Chui Neighbourhood Elderly Centre, Jockey Club CADENZA Hub, Ho Hong Neighbourhood Centre for Senior Citizens, and Heng On Social Centre for the Elderly) for their assistance in the recruitment process.

\section{REFERENCES}

Artero, S., Tierney, M. C., Touchon, J., and Ritchie, K. (2003). Prediction of transition from cognitive impairment to senile dementia: a prospective, longitudinal study. Acta. Psychiatr. Scand. 107, 390-393. doi: 10.1034/j.1600-0447.2003. 00081.x

Bowen, J., Teri, L., Kukull, W., McCormick, W., McCurry, S. M., and Larson, E. B. (1997). Progression to dementia in patients with isolated memory loss. Lancet 349, 763-765. doi: 10.1016/S0140-6736(96)08256-6

Chan, A. S., and Poon, M. W. (1999). Performance of 7- to 95-year-old individuals in a Chinese version of the category fluency test. J. Int. Neuropsychol. Soc. 5, 525-533. doi: 10.1017/S135561779956606X

Chan, A. S., Choi, M. K., and Salmon, D. P. (2001). The effects of age, education, and gender on the Mattis Dementia Rating Scale performance of elderly Chinese and American individuals. J. Gerontol. Psychol. Sci. 56, 356-363. doi: 10.1093/geronb/56.6.P356

Chan, A. S., Choi, A., Chiu, H., and Lam, L. (2003a). Clinical validity of the Chinese version of Mattis Dementia Rating Scale in differentiating dementia of Alzheimer's type in Hong Kong. J. Int. Neuropsychol. Soc. 9, 45-55. doi: 10.1017/S1355617703910058

Chan, A. S., Shum, D., and Cheung, R. W. (2003b). Recent development of cognitive and neuropsychological assessment in Asian countries. Psychol. Assess. 15, 257267. doi: 10.1037/1040-3590.15.3.257

Chan, A. S., Ho, Y. C., Cheung, M. C., Albert, M. S., Chiu, F. K., and Lam, C. W. (2005). Association between mind-body and cardiovascular exercises and memory in older adults. J. Am. Geriatr. Soc. 53, 1754-1760. doi: 10.1111/j.15325415.2005.53513.x

Chan, A. S. (2006). Hong Kong List Learning Test, 2nd Edn. Hong Kong: Department of Psychological and Integrative Neuropsychological Rehabilitation Center.

Chan, A. S. (2010). The Shaolin Chanwuyi: A Chinese Chan Buddhism. Hong Kong: Chanwuyi Publishing.

Chan, A. S. (2013). Contemporary Application of Shaolin Medicine: Dejian MindBody Intervention, 5th Edn. Hong Kong: Chanwuyi Publishing [in Chinese].

Chan, A. S., Sze, S. L., and Shi, D. (2008). Traditional Chinese mind-body exercises improve self control ability of an adolescent with Asperger's disorder. J. Psychol. Chin. Soc. 9, 225-239.

Chan, A. S., Sze, S. L., Cheung, M. C., Lam, J. M. K., and Shi, D. (2009). Dejian mindbody intervention improves the functioning of a patient with chronic epilepsy: a case report. Cases J. 2, 9080. doi: 10.1186/1757-1626-2-9080

Chan, A. S., Sze, S. L., Cheung, M. C., Han, Y. M. Y., Leung, W. W. M., and Shi, D. (2011a). Dejian mind-body intervention improves the cognitive functions of 
a child with autism. Evid. Based Complement. Alternat. Med. 2011:549254. doi: $10.1155 / 2011 / 549254$

Chan, A. S., Cheung, M. C., Tsui, W. J., Sze, S. L., and Shi, D. (2011b). Dejian mind-body intervention on depressive mood of community-dwelling adults: a randomized controlled trial. Evid. Based Complement. Alternat. Med. 2011:473961. doi: 10.1093/ecam/nep043

Chan, A. S., Cheung, M. C., Sze, S. L., Leung, W. W. M., and Shi, D. (2011c). Shaolin Dan Tian breathing fosters relaxed and attentive mind: a randomized controlled neuroelectrophysiological study. Evid. Based Complement. Alternat. Med. 2011:180704. doi: 10.1155/2011/180704

Chan, A. S., Wong, Q. Y., Sze, S. L., Kwong, P. P. K., Han, Y. M. Y., and Cheung, M. C. (2012a). A Chinese Chan-based mind-body intervention for patients with depression. J. Affect. Disord. 142, 283-289. doi: 10.1016/j.jad.2012.05.018

Chan, A. S., Wong, Q. Y., Sze, S. L., Kwong, P. P. K., Han, Y. M. Y., and Cheung, M. C. (2012b). A Chinese Chan-based mind-body intervention improves sleep on patients with depression: a randomized controlled trial. Sci. World J. 2012:235206. doi: 10.1100/2012/235206

Chan, A. S., Sze, S. L., Han, Y. M. Y., and Cheung, M. C. (2012c). A Chan dietary intervention enhances executive functions and anterior cingulate activity in autism spectrum disorders: a randomized controlled trial. Evid. Based Complement. Alternat. Med. 2012:262136. doi: 10.1155/2012/262136

Chan, A. S., Sze, S. L., Siu, N. Y., Lau, E. M., and Cheung, M. C. (2013a). A Chinese mind-body exercise improves self-control of children with autism: a randomized controlled trial. PLoS ONE 8:e68184. doi: 10.1371/journal.pone.0068184

Chan, A. S., Han, Y. M. Y., Sze, S. L., Wong, Q. Y., and Cheung, M. C. (2013b) A randomized controlled neurophysiological study of a Chinese Chan-based mind-body intervention in patients with major depressive disorder. Evid. Based Complement. Alternat. Med .2013:812096. doi: 10.1155/2013/812096

Chan, A. S., Han, Y. M., and Cheung, M. C. (2014). "Chinese Chan-based prospective neuropsychological intervention for autistic children," in The Comprehensive Guide to Autism, eds V. B. Patel, V. R. Preedy, and C. R. Martin (Berlin Heidelberg: Springer-Verlag).

Chen, P., Ratcliff, G., Belle, S. H., Cauley, J. A., DeKosky, S. T., and Ganguli, M. (2000). Cognitive tests that best discriminate between presymptomatic $\mathrm{AD}$ and those who remain nondemented. Neurology 55, 1847-1853. doi: 10.1212/WNL.55. 12.1847

Choi, J., and Twamley, E. W. (2013). Cognitive rehabilitation therapies for Alzheimer's disease: a review of methods to improve treatment engagement and self-efficacy. Neuropsychol. Rev. 23, 48-62. doi: 10.1007/s11065-0139227-4

Clare, L., and Woods, R. T. (2004). Cognitive training and cognitive rehabilitation for people with early-stage Alzheimer's disease: a review. Neuropsychol. Rehabil. 14, 385-401. doi: 10.1080/09602010443000074

Clark, F., Jackson, J., Carlson, M., Chou, C. P., Cherry, B. J., Jordan-March, M., etal. (2012). Effectiveness of a lifestyle intervention in promoting the well-being of independently living older people: results of the Well Elderly 2 randomised controlled trial. J. Epidemiol. Commun. Health 66, 782-790. doi: 10.1136/jech.2009.099754

D’Elia, L. F., Satz, P., Uchiyama, C., and White, T. (1996). Color Trails Test. Professional manual. Odessa, FL: Psychological Assessment Resources.

Dickinson, H. O., Mason, J. M., Nicolson, D. J., Campbell, F., Beyer, F. R., Cook, J. V., et al. (2006). Lifestyle interventions to reduce raised blood pressure: a systematic review of randomized controlled trials. J. Hypertens. 24, 215-233. doi: 10.1097/01.hjh.0000199800.72563.26

Edwards, J. D., Wadley, V. G., Myers, R. S., Roenker, D. L., Cissell, G. M., and Ball, K. K. (2002). Transfer of a speed of processing intervention to near and far cognitive functions. Gerontology 48, 329-340. doi: 10.1159/000065259

Gauthier, S., Reisberg, B., Zaudig, M., Petersen, R. C., Ritchie, K., Broich, K., et al. (2006). Mild cognitive impairment. Lancet 367, 1262-1270. doi: 10.1016/S01406736(06)68542-5

Hulette, C. M., Welsh-Bohmer, K. A., Murray, M. G., Saunders, A. M., Mash, D. C., and Mcintyre, L. M. (1998). Neuropathological and neuropsychological changes in "normal" aging: evidence for preclinical Alzheimer disease in cognitively normal individuals. J. Neuropathol. Exp. Neurol. 57, 1168-1174. doi: 10.1097/00005072-199812000-00009

Jacobson, N. S., and Truax, P. (1991). Clinical significance: a statistical approach to defining meaningful change in psychotherapy research. J. Consult. Clin. Psychol. 59, 12-19. doi: 10.1037/0022-006X.59.1.12
Lee, H. C. B., Chiu, H. F. K., Wing, Y. K., Chi, M. L., Po, K. K., and Chung, D. W. S. (1993). Chinese elderly and the GDS short form: a preliminary study. J. Aging Ment. Health 14, 37-42.

Lifestyle Medicine. (2013). Evidence Review: American College of Preventive Medicine (online). Available at: http://www.acpm.org/?LMI_History (accessed August 20, 2013).

Mahncke, H. W., Connor, B. B., Appelman, J., Ahsanuddin, O. N., Hardy, J. L., Wood, R. A., et al. (2006). Memory enhancement in healthy older adults using a brain plasticity-based training program: a randomized, controlled study. Proc. Natl. Acad. Sci. U.S.A. 103, 12523-12528. doi: 10.1073/pnas.06051 94103

McAvinue, L. P., Golemme, M., Castorina, M., Tatti, E., Pigni, F. M., Salomone, S., etal. (2013). An evaluation of a working memory training scheme in older adults. Front. Aging Neurosci. 5:20. doi: 10.3389/fnagi. 2013.00020

McGregor, K. M., Nocera, J. R., Sudhyadhom, A., Patten, C., Manini, T. M., Kleim, J. A., et al. (2013). Effects of aerobic fitness on aging-related changes of interhemispheric inhibition and motor performance. Front. Aging Neurosci. 5:66. doi: 10.3389/fnagi.2013.00066

Papp, K. V., Walsh, S. J., and Snyder, P. J. (2009). Immediate and delayed effects of cognitive interventions in healthy elderly: a review of current literature and future directions. Alzheimers Dement. 5, 50-60. doi: 10.1016/j.jalz.2008. 10.008

Petersen, R. C., Smith, G. E., Waring, S. C., Ivnik, R. J., Tangalos, E. G., and Kokmen, E. (1999). Mild cognitive impairment: clinical characterization and outcome. Arch. Neurol. 56, 303-308. doi: 10.1001/archneur. 56.3.303

Petersen, R. C., Stevens, J. C., Ganguli, M., Tangalos, E. G., Cummings, J. L., and DeKosky, S. T. (2001). Practice parameter: early detection of dementia: mild cognitive impairment (an evidence-based review): report of the Quality Standards Subcommittee of the American Academy of Neurology. Neurology 56, 1133-1142. doi: 10.1212/WNL.56.9.1133

Rasmunsson, D. X., Rebok, G. W., Bylsma, F. W., and Brandt, J. (1999). Effects of three types of memory training in normal elderly. Aging Neuropsychol. C. 6, 56-66. doi: 10.1076/anec.6.1.56.790

Ruscheweyh, R., Willemer, C., Krüger, K., Duning, T., Warnecke, T., Sommer, J., et al. (2011). Physical activity and memory functions: an interventional study. Neurobiol. Aging 32, 1304-1319. doi: 10.1016/j.neurobiolaging.2009. 08.001

Smith, G. E., Housen, P., Yaffe, K., Ruff, R., Kennison, R. F., Mahncke, H. W., et al. (2009). A cognitive training program based on principles of brain plasticity: results from the Improvement in Memory with Plasticity-based Adaptive Cognitive Training (IMPACT) study. J. Am. Geriatr. Soc. 57, 594-603. doi: 10.1111/j.1532-5415.2008.02167.x

Tierney, M. C., Szalai, J. P., Snow, W. G., Fisher, R. H., Nores, A., Nadon, G., etal. (1996). Prediction of probable Alzheimer's disease in memoryimpaired patients: a prospective longitudinal study. Neurology 46, 661-665. doi: 10.1212/WNL.46.3.661

Yu, R., Woo, J., Chan, A. S., and Sze, S. L. (in press). A Chinese Chan-based mind-body intervention improves psychological well-being and physical health of community-dwelling elderly: a pilot study. Clin. Interv. Aging.

Wechsler, D. (2005). Wechsler Memory Scale III (Chinese): Manual. Taiwan: Psychological Corporation.

Willis, S. L., Tennstedt, S. L., Marsiske, M., Ball, K., Elias, J., Koepke, K. M., et al. (2006). Long-term effects of cognitive training on everyday functional outcomes in older adults. JAMA 296, 2805-2814. doi: 10.1001/jama.296. 23.2805

Wise, E. A. (2004). Methods for analyzing psychotherapy outcomes: a review of clinical significance, reliable change, and recommendations for future directions. J. Pers. Assess. 82, 50-59. doi: 10.1207/s15327752 jpa8201_10

Witte, A. V., Fobker, M., Gellner, R., Knecht, S., and Flöel, A. (2009). Caloric restriction improves memory in elderly humans. Proc. Natl. Acad. Sci. U.S.A. 106, 1255-1260. doi: 10.1073/pnas.0808587106

Conflict of Interest Statement: The authors declare that the research was conducted in the absence of any commercial or financial relationships that could be construed as a potential conflict of interest. 
Received: 24 December 2013; paper pending published: 19 February 2014; accepted: 03 March 2014; published online: 26 March 2014.

Citation: Chan AS, Sze SL, Woo J and Yu RH (2014) A Chinese Chan-based lifestyle intervention improves memory of older adults. Front. Aging Neurosci. 6:50. doi: 10.3389/fnagi.2014.00050

This article was submitted to the journal Frontiers in Aging Neuroscience.
Copyright (c) 2014 Chan, Sze, Woo and Yu. This is an open-access article distributed under the terms of the Creative Commons Attribution License (CC BY). The use, distribution or reproduction in other forums is permitted, provided the original author(s) or licensor are credited and that the original publication in this journal is cited, in accordance with accepted academic practice. No use, distribution or reproduction is permitted which does not comply with these terms. 